\title{
Idiopathic nephrotic syndrome
}

INSERM

\section{Source}

INSERM. (1999). Orphanet: an online rare disease and orphan drug data base. Idiopathic nephrotic syndrome. ORPHA:357502

A rare primary glomerular group of diseases characterized by the triad of edema, massive, or nephrotic-range, proteinuria and hypoalbuminemia, for which there is no known cause. Depending on response to treatment, disease is distinguished into steroidsensitive nephrotic syndrome (SSNS) and steroid-resistant nephrotic syndrome (SRNS), with the latter being further divided, depending on occurrence, into familial or sporadic forms. 